\title{
Theoretical Study of Physico-mechanical Response of Permeable Fluid-Saturated Materials Under Complex Loading Based on the Hybrid Cellular Automaton Method
}

\section{Andrey V. Dimaki and Evgeny V. Shilko}

\begin{abstract}
We give a brief description of the results obtained by Prof. Sergey G. Psakhie and his colleagues in the field of theoretical studies of mechanical response, including fracture, of permeable fluid-saturated materials. Such materials represent complex systems of interacting solid and liquid phases. Mechanical response of such a medium is determined by processes taking place in each phase as well as their interaction. This raised a need of developing a new theoretical approach of simulation of such media-the method of hybrid cellular automaton that allowed describing stress-strain fields in solid skeleton, transfer of a fluid in crack-pore volume and influence of fluid pressure on the stress state of the solid phase. The new method allowed theoretical estimation of strength of liquid-filled permeable geomaterials under complex loading conditions. Governing parameters controlling strength of samples under uniaxial loading and shear in confined conditions were identified.
\end{abstract}

Keywords Hybrid cellular automaton · Poroelasticity $\cdot$ Strength $\cdot$ Permeability Fluid-saturated materials

\section{Introduction}

Many natural and man-made materials and media, such as permeable rocks (including coal and oil bearing strata) [1-4], bone tissue [5], filtering materials [6], endoprostheses [7] and other, are fluid-saturated porous or cracked porous media. The mechanical response of fluid-saturated permeable materials exhibits some features that differentiate them from solid composite materials in which phases also have different elastic and rheological characteristics. These features are associated with the ability of a liquid/gaseous phase to redistribute in cracks and pores of an enclosing material. As a result of the redistribution, mean stress can either level off in different regions of the material or strongly oscillate due to dilatancy and filling of new discontinuities or due to fluid exchange with the surrounding medium.

\footnotetext{
A. V. Dimaki ( $\varangle) \cdot$ E. V. Shilko

Institute of Strength Physics and Materials Science SB RAS, Tomsk, Russia

e-mail: dav18@yandex.ru 
At present, numerous computational methods do exist describing the mechanical behavior of a continuous medium within a certain scale level (finite difference, boundary element, and cellular automata methods [8], etc.). The most popular among them are the finite element method and different variations of the particle method (molecular dynamics, discrete elements, movable cellular automaton (MCA) [911] etc.). Distribution of gas and liquid in various porous media is simulated using such modern and intensively developing methods as the lattice Boltzmann method [12] and methods based on the solution of the Navier-Stokes equations on a finitedifference grid. However, the description of a multiscale contrast medium containing interacting solid, liquid, and gas phases within a common approach meets problems connected with description of interrelation between solutions of equations describing the behavior of each phase. Solution of this problem can be found in development of new methods and approaches giving explicit consideration to the multiscale and behavioral peculiarities of studied objects. The most effective among them are methods based on the principles of physical mesomechanics that considers a solid as a multilevel system [13].

Professor Sergey Psakhie founded the method of hybrid cellular automaton (HCA) and was the leader of numerous pioneering works devoted to theoretical study of multiscale and multiphase media using this method. His ideas allowed for solution of many fundamental and practical problems connected with behavior of geological media under complex dynamic loading. Among these media are coal beds filled with gas, porous permeable materials filled with liquid, weakly connected boundaries (shear bands) in geomaterials with gradients of permeability under shear loading conditions.

\section{Brief Description of the Hybrid Cellular Automaton Method}

One possible theoretical approach to the study of fluid-saturated permeable materials is to use coupled models that account for the following important aspects of such systems behavior: (1) relation of solid skeleton deformation with volume and porosity variation in the pore and crack volume; (2) relation between pore pressure and stress in the enclosing volume of a solid; (3) fluid redistribution in the pore and crack volume. The most famous representatives of this approach are analytical macroscopic models of poroelasticity whose theoretical basis was first discussed by Biot [14, 15]. They were further developed by taking into account a range of scales in real materials, damage accumulation, dilatancy and their influence on the skeleton elastic properties and pore fluid pressure [16-22].

The computational methods based on a discrete representation of the medium are widely used to describe media where fracture on different scales is a factor determining a mechanical response. A well-known representative of this family of models is the discrete element method (DEM) in which the modeled material is 
represented as an ensemble of interacting finite-size particles [23-32]. Its major advantages result from the ability of discrete elements to change their surroundings, which is crucial for simulation of complex phenomena such as contact interaction, cracking and fragmentation of solids, flow of granulated media and other.

Various explicit DEMs use different approximations to describe strain distribution within the discrete element volume and the influence of element shape/geometry on its kinematics and interaction with the surroundings [29]. A common approach to description of an element shape is an approximation of an equivalent circular disc or sphere [23, 28, 29]. Further we consider equiaxed or nearly equiaxed elements in approximation of equivalent circular discs and use the term "discrete element" to mention the given simplified representation of an element shape. The given approach has a simple mathematical formulation and apparent advantages in modelling deformation and fracture.

Further development of the DEM formalism expanded its application to a wider range of spatial and structural scales [33-38] and yielded a coupled numerical method for studying permeable media on the meso- and macroscale levels. This coupled numerical method was called the hybrid cellular automaton method. In the HCA method the mechanical response of the enclosing solid is described by the MCA method [39, 40]; fluid filtration and diffusion in cracks and pores of the solids (taken into account implicitly) are described by a finite difference method.

The numerical HCA method is based on separation of the problem into two parts: (1) description of the mechanical behavior of the enclosing solid (skeleton), and (2) description of a fluid transfer in the filtration volume of the solid represented by a system of connected channels, pores, cracks and other discontinuities. Depending on structural features of a considered permeable material and on the simulated scale, the dimensions of discrete elements can be much larger than the linear dimensions of discontinuities in the solid, can be comparable to them or smaller. The influence of "micropores" in the solid skeleton (i.e., pores, channels and other discontinuities whose typical size is smaller than discrete element size) on the mechanical properties and response of a discrete element is accounted for explicitly. Additionally, the MCA method is used to solve the problem of filtration fluid transfer in the network of connected "micropores" of the enclosing solid. Fluid mass transfer between "micropores" and "macropores", which are considered as regions between spaced and noninteracting discrete elements, is calculated using a finer grid embedded in a laboratory coordinate system (Fig. 1a). The same grid is used to calculate "macropore" volumes (Fig. 1b).

Within the MCA formalism local failure is modelled by changing the state of a pair of interacting elements from linked (or bonded) to unlinked. In simulations described below we apply the modified Drucker-Prager failure criterion taking into account local pore fluid pressure:

$$
\sigma_{D P}=0.5(\lambda+1) \sigma_{e q}+1.5(\lambda-1)\left(\sigma_{\text {mean }}+b P_{\text {pore }}\right)=\sigma_{c}
$$

where $\lambda=\sigma_{c} / \sigma_{t}^{i}$ is the ratio of compressive $\sigma_{c}$ to tensile $\sigma_{t}$ strength, $\sigma_{e q}$ - equivalent stress, $b$ is the dimensionless coefficient determining a contribution of a fluid pore 


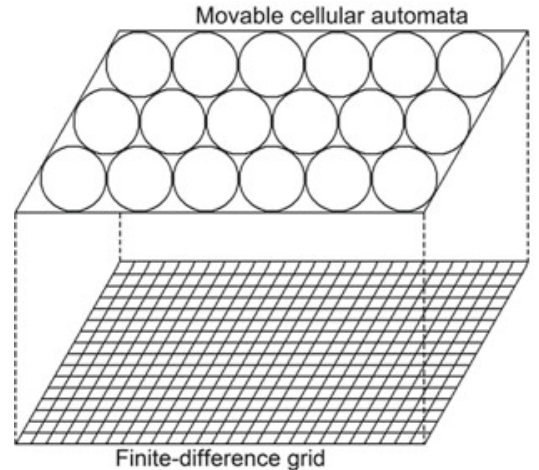

a)

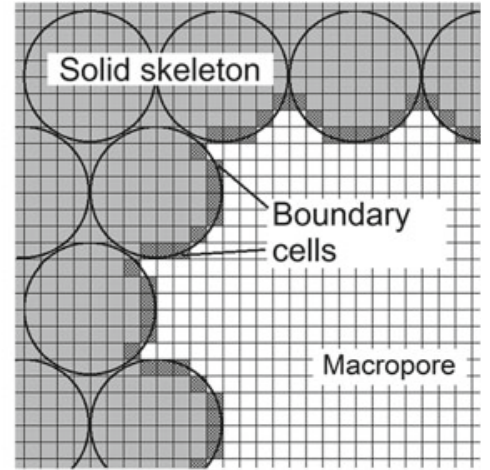

b)

Fig. 1 Layers of discrete elements (movable cellular automata) and finite-difference grid (a); grid cells at a boundary between solid skeleton and macropores (b)

pressure into mean stress $\sigma_{\text {mean }}$ and $P_{\text {pore }}$ is the fluid pore pressure. A comprehensive description of details of the numerical implementation of hybrid cellular automaton method is given in [41, 42].

\section{Strength of Porous Fluid-Filled Samples Under Uniaxial Loading: A Competition Between Compression and Fluid Filtration}

The developed model was applied to study a mechanical response of porous elasticbrittle samples with water-filled filtration volume under uniaxial compression. The samples were fixed between a matrix (at the bottom) and a punch (at the top) that moved downwards and compressed the sample with constant velocity $V_{y}$. The compression direction coincided with the vertical sample axis. The problem was solved in a 2D statement in the plane stress approximation. The sample structure was assumed to be homogeneous, without pores and inclusions. The sample height was $H=0.1 \mathrm{~m}$ and width was $W=0.05 \mathrm{~m}$. Numerical experiments were performed using the following parameters of the model material: $K=37.5 \mathrm{GPa}, K_{s}=107 \mathrm{GPa}$, $G=5.77 \mathrm{GPa}, \rho=2000 \mathrm{~kg} / \mathrm{m}^{3}, \lambda=7, \sigma_{c}=70 \mathrm{MPa}, K_{f l}=2.2 \mathrm{GPa}$ (see details in $[41,42])$. Initial value of material porosity was $\varphi_{0}=0.1$.

Two hypotheses of micropore distribution in the solid skeleton were considered with regard to the influence of pore pressure on sample strength.

1. Micropores are distributed homogeneously. Their size is much smaller than the characteristic size of damages and cracks formed during fracture. In this case, we assume that the effect of pore fluid on skeleton strength is governed by the porosity 
value and is taken into account by subsequent determination of coefficient $b$ in failure criterion (1): $b=\varphi_{0}$.

2. Micropores are distributed inhomogeneously, and damages in the material are formed through coalescence of several micropores. In this case, the effect of pore fluid on skeleton strength is directly defined by the pore pressure value: $b=1$.

The simulation results revealed that pore fluid pressure exerts a strong influence on the mechanical response of brittle porous samples. Other factors that significantly influence the sample strength are the loading rate, characteristic filtration channel diameter (this quantity, along with open porosity, determines material permeability) and geometrical dimensions of the sample.

Fluid pressure in pores of a solid under uniaxial compression is governed by two competing processes: (1) solid skeleton deformation accompanied by pore volume reduction and by pore pressure increase (and, consequently, increasing influence of fluid on the stress state of the skeleton); (2) fluid discharge to the environment through the lateral faces of the sample, due to which pore pressure decreases and the influence of fluid on the stress state of the skeleton also decreases.

A balance of the mentioned two processes is governed by permeability coefficient. At low sample permeability fluid outflow from the sample does not compensate fluid density increase during pore deformation. As a result, fluid pressure in the filtration volume constantly increases in the course of deformation, due to which the effective sample strength decreases. At large permeability, the rate of fluid outflow from the sample is sufficient to reduce pore pressure to zero. The influence of fluid on the stress state of such samples is nearly absent and their strength tends to the strength of a "dry" sample. Between these "limiting" cases the rate of fluid pressure decrease caused by fluid outflow from the sample is comparable to the rate of fluid pressure increase due to solid skeleton compression.

Sample strength is also determined by fluid pressure distribution in the pore volume across the sample cross section. This distribution depends on the ratio of sample width $W$ to its height $H$. The material volume which is adjacent to the lateral face and through which fluid escapes to the environment decreases with the increasing sample width. Correspondingly, the specific amount and pressure of the fluid retained in pores at the beginning of fracture increases with the growing $W / H$ ratio. This leads to an unexpected conclusion: other factors being equal, the strength of a water-saturated sample of larger width appears to be lower than the strength of a "narrower" sample.

As above, the strength of fluid-saturated permeable samples is governed by the competition of mechanical deformation under applied external load and fluid discharge from the pore space to the environment. To reveal general mechanisms of this competition, we studied the effect of loading rate on uniaxial compressive strength of fluid-saturated samples. Uniaxial compression of fluid-saturated samples with different values of $d_{c h}$ was modeled for different loading rates $V_{y}$. The initial pore fluid pressure $P_{\text {init }}$ was assumed to be zero (pore volume of samples was completely filled with fluid at atmospheric pressure). 


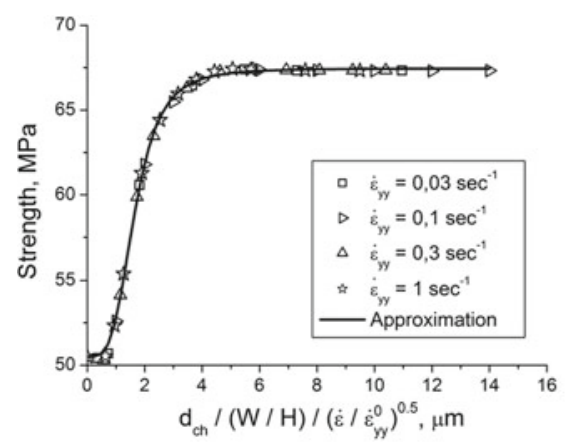

a)

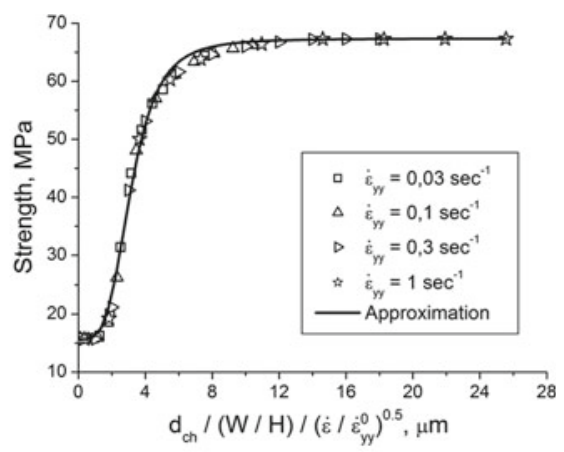

b)

Fig. 2 Generalized dependence of water-saturated sample strength on filtration channel diameter at different loading rates: a approximation of homogeneous micropore distribution $(b=\varphi=0.1)$; b approximation of relatively large micropores whose evolution leads to macrocrack formation $(b=$ 1). Initial fluid pressure in solid skeleton pores is $P_{\text {init }}=P_{0}$. In all calculations the aspect ratio of samples was $W / H=0.5$

Figure 2 gives the dependences of sample strength on the characteristic filtration channel diameter at different strain rates. The value of parameter $b$ that determines the pore pressure contribution to failure criterion (1) strongly affects the strength of fluid-saturated samples. For example, within the approximation of homogeneous micropore distribution in the solid skeleton $(b=\varphi=0.1$, Fig. 2a) the pore pressure contribution is rather low (maximum decrease in sample strength does not exceed $25 \%)$. Within the second approximation ( $b=1$, Fig. $2 \mathrm{~b})$ the strength of watersaturated samples at low permeability values can decrease several-fold. The strength dependence flattens out in this case due to a stronger influence of residual fluid in the pore space on sample strength.

Analysis of the obtained dependences of sample strength on the effective filtration channel diameter $d_{c h}$ at different loading rates and different $W / H$ ratios revealed that they can be reduced to a single dependence of strength on the reduced effective filtration channel diameter:

$$
d_{c h} /\left((W / H) \sqrt{\dot{\varepsilon}_{y y} / \dot{\varepsilon}_{y y}^{0}}\right)
$$

where $\dot{\varepsilon}_{y y}=V_{y} / H$ is the strain rate, and $\dot{\varepsilon}_{y y}^{0}$ is the scale multiplier that has the dimension of strain rate. The curves shown in Fig. 2 are plotted in these variables $\left(\dot{\varepsilon}_{y y}^{0}\right.$ was assumed to be equal to $\left.1 \mathrm{~s}^{-1}\right)$.

It is known that the processes whose occurrence is governed by the competition of several factors or phenomena (e.g., biological population growth, etc.) are often described by a logistic function [43]. Based on the above assumption about the decisive role of the competition between pore pressure increase and fluid outflow from the sample, we used the following logistic function to approximate the dependence of 
strength of uniaxially compressed water-saturated samples on the reduced effective filtration channel diameter:

$$
\sigma_{c}\left(d_{c h}, \dot{\varepsilon}_{y y}\right)=\sigma_{c}^{\min }+\frac{\sigma_{c}^{0}-\sigma_{c}^{\min }}{1+\left(d_{c h} /\left(d_{0}(W / H) \sqrt{\dot{\varepsilon}_{y y} / \dot{\varepsilon}_{y y}^{0}}\right)\right)^{p}}
$$

where $\sigma_{c}^{0}$ is the sample strength under uniaxial compression in the absence of fluid in the pore space, $\sigma_{c}^{\min }$ is the water-saturated sample strength in the absence of fluid mass transfer, $d_{0}$ is the parameter of the approximating function having the dimension of distance, $\dot{\varepsilon}_{y y}$ is the axial strain rate of the sample, and $p$ is the exponent. The parameters of Eq. (3) are defined by the elastic moduli of the solid skeleton and fluid, fluid viscosity, porosity value and so on. As one can see, logistic function (3) allows approximation the numerically calculated data given in Fig. 2 with good accuracy (at $d_{0}=1.62 \mu \mathrm{m}, p=3.7$ for the curve in Fig. $2 \mathrm{a}$ and at $d_{0}=3.07 \mu \mathrm{m}, p$ $=4$ for the curve in Fig. $2 b$ ).

In order to generalize the results it is useful to take reduced material permeability $k$ as the parameter determining fluid filtration rate:

$$
\frac{d_{c h}}{\sqrt{\frac{\dot{\varepsilon}_{y y}}{\dot{\varepsilon}_{y y}^{0}} \frac{W^{2}}{H^{2}}}} \rightarrow \sqrt{\frac{k}{\dot{\varepsilon}_{y y}} \frac{H^{2}}{W^{2}} \frac{\dot{\varepsilon}_{y y}^{0}}{\varphi_{0}}}
$$

Within this formulation, parameter (2) and approximating function (3) take a more general meaning and can be applied to permeable materials with different structure of filtration volume. Complex relations between the parameters characterizing the mechanical response of the solid skeleton, physical and mechanical properties of fluid and its filtration redistribution dynamics in the system of pores define the nonlinear dependence of sample strength on a combination of these parameters and necessitates the application of numerical methods to study the mechanical response of fluidsaturated porous materials.

\section{Influence of Pore Fluid Pressure and Material Dilation on Strength of Shear Bands in Fluid-Saturated Rocks}

A range of laboratory and full-scale geological and geophysical research suggests that irreversible deformation in rock samples and rock massifs is strongly localized in shear bands at different scales, the largest of which are tectonic faults [44-46]. These narrow zones not only determine the compliance of rocks in the form of localized relative shear displacements of structural blocks, but control seismic activity of rock massifs. The latter explains the current interest in the mechanical properties of fault zones and the rapid increase in the number of published works in this area. 
One of the key mechanical properties is the maximum (or peak) strength of the fault zone under given stress and confinement conditions. Reaching the maximum strength corresponds to a change in the response of the shear band from pervasive strain (strain hardening stage) to strain localization (strain softening stage) [47, 48]. Maximum shear strength estimation of fault zones is both a fundamental and practical problem widely discussed in fault and rock mechanics [49-52].

The conditions for onset of pervasive inelastic strain and subsequent reaching of the maximum strength of shear bands (including faults) are mainly affected by the pore structure and pore fluid pressure. The pore pressure dynamics is controlled by two interrelated processes [53-63]: (1) fluid flow and (2) pore volume change. The pervasive inelastic deformation of rock is often accompanied by its dilatancy [64-66]. The volume of the connected crack-pore space increases during pervasive shear deformation of the shear band, that leads to decrease of local pore pressure. The reduction of fluid pressure reduces the intensity of the relaxation processes associated with the formation of new discontinuities and coalescence of existing ones. This effect is called dilatancy hardening [54]. In turn, fluid inflow is able to compensate for the pore pressure drop and reduce the effect of strain hardening [63, 67]. The ratio of fluid flow rate to strain rate (which governs the dilation rate) determines the specific value of shear strength of shear bands. Note that the influence of the competition between dilatancy and fluid flow on shear strength is strongly pronounced for shear bands surrounded by material blocks with a similar permeability to that of the shear band gouge. This particularly corresponds to healed (consolidated) faults where the difference in the porosity and permeability of the principal slip zone (of width 1$10 \mathrm{~cm}$ ) and surrounding periphery zone (up to several meters wide) is much less pronounced than in faults with a mature zone of unconsolidated gouge [68].

Conventionally, the effect of pore fluid on the maximum shear strength (hereinafter referred to as strength) of shear bands, including fault zones, has been studied for limiting modes of deformation (very slow and very fast) that correspond to drained and undrained hydrological conditions [69] due to the fact that limiting modes correspond to the long-term creep and short-term dynamic modes of deformation of fault regions. Numerous experimental and theoretical works, starting with the classical work of Brace and Martin [69], show that the strength of permeable rock samples can increase significantly (up to 30-50\%) in transition from the drained to undrained condition $[67,70,71]$. This is explained by the limitation of fluid inflow to the increasing pore space of the incipient shear band and corresponding inhibition of pore pressure drop recovery.

There is still no unambiguous understanding of how the strength of a shear band in the depth of constrained permeable rock massif changes in the transition region between the undrained and drained conditions. We studied the nature and functional form of the dependence of the shear band strength on the ratio of shear strain rate to fluid flow rate under constrained conditions corresponding to faults in rock massifs. The study was performed by numerical modelling of the shear deformation of a fluid-saturated permeable shear band using the discrete element method.

Consider a model sample consisting of two blocks separated by an interfacial layer (shear band) in the plane strain approximation (Fig. 3). The blocks mimic regions of 


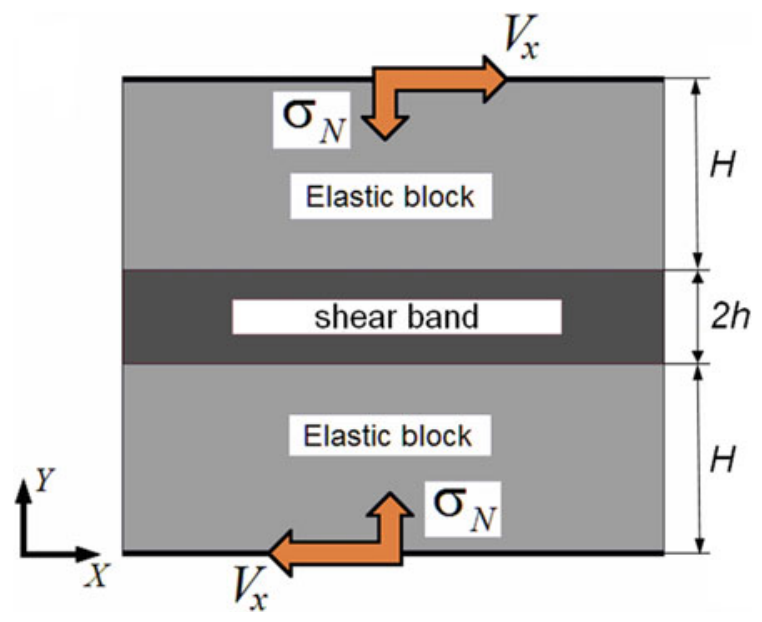

Fig. 3 Sample structure and loading scheme for modelling of constrained shear of a porous fluidsaturated shear band surrounded by porous fluid-saturated blocks

the medium adjacent to the shear band, which are less damaged than the shear band and therefore deform elastically under the considered loading conditions. The shear band of width $2 h$ is a layer of an elastic-plastic dilatant material, which simulates the layer of consolidated gouge in principal slip zones of faults [47, 72]. The width of the model blocks was $H=20 h$. We used the following reference values of widths of the shear band and blocks: $2 h \approx 1.5 \mathrm{~cm}, H=15 \mathrm{~cm}$. The shear band and blocks were assumed to be permeable and fluid saturated.

The model shear band with surrounding fragments of blocks was numerically simulated by the discrete element method using a fully coupled macroscopic model of fluid-saturated porous brittle materials [33-41]. Within the model discrete elements simulating parts of the shear band and surrounding blocks are treated as porous and permeable. The effect of the fluid contained in the crack-pore volume of a discrete element on its stress state is described based on Biot linear model of poroelasticity $[14,15]$. The inelastic behavior of the permeable brittle material of the discrete element is described using a plastic flow model of rocks with a non-associated flow law and the Mises-Schleicher yield criterion (Nikolaevsky's model) [16]. The elastic characteristics of the shear band and blocks were assumed to be similar and corresponded to typical values for sandstones with a porosity of 10-15\% (Young's modulus $E=15 \mathrm{GPa}$, Poisson's ratio $v=0.3$ ). The material of discrete elements modelling the blocks was treated as elastic-brittle and high-strength. The material of discrete elements modelling the shear band was a model elastic-plastic material with linear hardening with the following plasticity and strength parameters: $\beta=$ $0.57, \Lambda=0.36, Y=10.84 \mathrm{MPa}$ (this corresponds to a yield stress of $28 \mathrm{MPa}$ under uniaxial compression), strain hardening modulus $\Pi=515 \mathrm{MPa}$, uniaxial compressive strength $\sigma_{c}=40 \mathrm{MPa}$, uniaxial tensile strength $\sigma_{t}=13.33 \mathrm{MPa}(\lambda=3)$. The 
calculations were carried out at an initial mean stresses $\sigma_{\text {mean }}^{0}$ below the brittle-ductile transition threshold (in this case, at $\sigma_{\text {mean }}^{0}<40 \mathrm{MPa}$ ).

The initial values of porosity $\left(\phi_{0}=0.1\right)$ and permeability $k_{0}$ of the shear band and the blocks were assumed to be equal. This approximation is consistent with the low gradients of porosity and permeability in central zones of healed (consolidated) faults. Initially, all interacting elements were linked that simulates a consolidated shear band.

We modelled constrained shear of the sample in the horizontal plane along the $X$ axis (Fig. 3). Periodic boundary conditions were specified on the lateral faces in the horizontal direction to simulate an infinitely long shear band. The sample was loaded in two stages. At the first stage, a normal load $\sigma_{N}$ was applied to the upper and lower sample faces. The initial fluid concentration in the pore space of the sample was chosen so as to create the specified pore pressure $P_{\text {pore }}^{0}$ in the sample. There was no plastic deformation in the sample by the end of the first loading stage. The stress and pore pressure distributions were homogeneous. At the second stage, the sample was subject to simple shear by applying constant tangential velocity $V_{x}$ and zero normal velocity (along the $Y$ axis) to the upper and lower faces to fulfil the constrained shear condition. The sample deformation proceeded until crack initiation in the shear band.

The described 2D system models a horizontal cross section of a healed fault between structural blocks of a rock massif at a certain depth. Note that the initial "horizontal" (in the $X Y$ plane) stresses in the given formulation of the problem exceed the "vertical" ones. This is consistent with experimental data indicating that horizontal stresses are considerably higher than vertical ones in regions with high deformation activity [73]. We used isolated conditions on the external surfaces of the sample (hydraulically isolated sample) that correspond to the hydrological conditions in the central regions of fault zones in the bulk of low permeable host rocks.

The simulation results showed that at high strain rates the magnitude of shear strength tends toward the upper limit, and at low strain rates-toward the minimal value. Such regularity was first observed by Brace and Martin [69], and then reported in numerous experimental and theoretical studies of confined compression of rock samples and shear loading of model fault zones (see e.g. [67]).

The reduction of shear strength from the upper to the lower limit with reduction of strain rate is not monotonous. At a certain intermediate strain rate the shear strength reaches a local minimum. Further reduction of strain rate leads to an increase of shear strength up to a local maximum. At even smaller strain rates the shear strength again decreases down to the lower limit. This result quantitatively agrees with recent experimental studies [74-76].

We varied the shear strain rate $\dot{\varepsilon}_{x y}=V_{x} /(H+h)$, the initial permeability of the blocks and the shear band $k_{0}$ (at fixed $\phi_{0}=0.1$ ), the dynamic fluid viscosity $\eta$, and system size $(2 H+2 h)$ within several orders of magnitude: $\dot{\varepsilon}_{x y}$ from $5 \times 10^{-4} \mathrm{~s}^{-1}$ to $1 \mathrm{~s}^{-1}, k_{0}$ from $10^{-18} \mathrm{~m}^{2}$ to $10^{-13} \mathrm{~m}^{2}, \eta$ from $2 \times 10^{-4} \mathrm{~Pa}$ s to $2 \times 10^{-2} \mathrm{~Pa}$ s (dynamic viscosity of water at room temperature is about $\left.10^{-3} \mathrm{~Pa} \mathrm{~s}\right),(2 H+2 h)$ from 15 to $150 \mathrm{~cm}$. We found that the parameter combination 


$$
A_{x y}=\frac{\dot{\varepsilon}_{x y} \eta(H+h)^{2}}{k_{0}}
$$

unambiguously determines the value of shear strength of the shear band for a given initial mean stress $\sigma_{\text {mean }}^{0}$, pore pressure $P_{\text {pore }}^{0}$, and ratio $h / H$. In other words, shear band zones have the same shear strength if they are characterized by the same value of $A_{x y}$, (even if the specific values of the parameters $k_{0}, \eta, \dot{\varepsilon}_{x y}$, and $h$ differ by orders of magnitude). The parameter $A_{x y}$ means the relation of strain rate to fluid flow rate.

Figure 4 shows a typical dependence of the shear strength $\tau_{c}$ of the modelled shear band on the parameter $A_{x y}$ for a hydraulically isolated system. Each point of the curve corresponds to a separate calculation at given values of $k_{0}, \eta, \dot{\varepsilon}_{x y}$ and $h$ (at $h / H=$ const, $\sigma_{N}=$ const, $P_{\text {pore }}^{0}=$ const $)$. The region $A_{x y} \rightarrow \infty$ (region I in Fig. 4) corresponds to combinations of $k, \eta, \dot{\varepsilon}_{x y}$ and $h$ where the fluid flow rate is extremely low compared to the rate of pore pressure change caused by pore volume variation. This corresponds to the hydrological conditions close to the undrained condition of the shear band. The region of low $A_{x y}$ values (region III in Fig. 4) corresponds to low shear rate, low fluid viscosity or high permeability of the blocks. In this region the fluid flow rate is relatively high, and the hydrological conditions for the shear band approach a fully drained (the pore pressure distribution in the sample is close to homogeneous during the entire course of deformation).

The curve shown in Fig. 4 has three characteristic regions where the change of strength is monotonous, that implies a presence of a dominant mechanism determining the direction of the change.

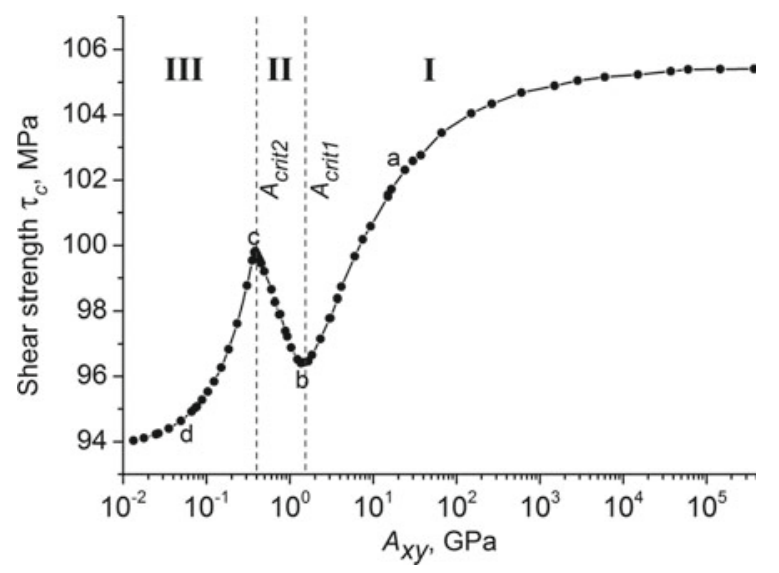

Fig. 4 A typical dependence of the shear strength $\tau_{c}$ of the shear band on the parameter $A_{x y}$ for a hydraulically isolated sample. Roman numerals I-III mark the curve regions corresponding to different behaviour modes of the fluid-saturated sample under shear loading. The values $A_{\text {critl }}$ and $A_{\text {crit } 2}$ correspond to the local minimum and maximum shear strength. The top and bottom faces of the sample are fixed in vertical direction. Lower $A_{x y}$ imply faster fluid flow or lower strain rate, higher $A_{x y}$ imply slower fluid flow or higher strain rate 
In region I the dominating mechanism lies in the decrease of the linear dimensions of the blocks due to fluid outflow to the shear band (poroelastic contraction). Decrease in the value of $A_{x y}$ is accompanied by the inflow of a large amount of fluid into the shear band and hence by reduction of the constraint imposed on the shear band by the compressed blocks (effective normal stiffness of the blocks decreases). This mechanism determines the decrease of the shear strength in region I as $A_{x y}$ decreases.

In region II the trend-determining mechanism is tied to the increase of the dilation rate of the shear band with decreasing value of $A_{x y}$ due to slowing of pore pressure reduction and maintaining nonzero pore pressure during most of the shear process. This mechanism provides an increase in the absolute value of effective mean stress in the sample and hence increase in the strength of the shear band in region II as $A_{x y}$ decreases.

In region III the trend-determining mechanism is linked to the fact that pore pressure in the shear band remains non-zero during the entire shear process. In this region pore pressure in the shear band is higher in the samples characterized by lower values of $A_{x y}$. Because of this fact an absolute value of effective mean stress in a shear band is also lower in the samples with lower $A_{x y}$. Decrease of an absolute value of effective mean stress leads to gradual decrease in the shear strength down to the absolute minimum at $A_{x y} \rightarrow 0$.

The described three parts of the curve $\tau_{c}\left(A_{x y}\right)$ have sigmoid profiles that is the result of the competition between shear band dilatancy and poroelastic contraction of the blocks due to fluid outflow. Analysis of the obtained result allowed formulating the following general dependence of the shear strength of constrained shear band zones on the parameter $A_{x y}$. The dependence is expressed as a sum of a constant and three sigmoid contributions:

$$
\tau_{c}=\tau_{0}+\frac{\tau_{1}}{1+\left(c_{1} A_{x y}\right)^{-p_{1}}}+\frac{\tau_{2}}{1+\left(c_{2} A_{x y}\right)^{p_{2}}}-\frac{\tau_{3}}{1+\left(c_{3} A_{x y}\right)^{p_{3}}},
$$

where $\tau_{1}, \tau_{2}$ and $\tau_{3}$ are the amplitudes of contributions of the three above-mentioned mechanisms to the shear strength, $c_{1}, c_{2}$ and $c_{3}$ are the inverse positions of the sigmoid midpoints, the exponents $p_{1}, p_{2}$ and $p_{3}$ determine the steepness of the sigmoid functions and $\tau_{0}$ is a constant contribution independent of the fluid flow dynamics.

The amplitudes of the contributions have the following physical meanings. The constant contribution $\tau_{0}$ is the strength of the shear band in the absence of fluid flow (or under high strain rate) at a constant value of normal force $\sigma_{N}$ applied to the upper and lower sample faces during the entire course of shear. Another three contributions are concerned with squeezing of the blocks due to shear band dilation in a mechanically constrained sample.

The specific parameter values of Eq. (6) depend on mechanical characteristics of the skeleton of shear band and blocks, bulk modulus of fluid, the $h / H$ ratio, initial mean stress $\sigma_{\text {mean }}^{0}$, fluid content in the blocks and hydrological conditions. 


\section{Conclusion}

We have presented the results of application of the hybrid numerical technique for theoretical study of deformation and fracture of fluid-saturated permeable materials and media. In the framework of this technique the simulated medium is considered as a superposition of two interdependent layers. One layer is represented by an ensemble of particles (simply deformable discrete elements), and the other-by a finite-difference grid. This approach combined with Biot's model of poroelasticity is suitable for studying complex and interrelated processes of solid skeleton deformation and fracture and fluid redistribution (mass transfer) in the pore volume.

Strength of water-saturated elastic-brittle samples under uniaxial compression significantly depends on fluid pressure in the pore space as well as on solid permeability, physical and mechanical properties of fluid, strain rate and sample dimensions. The numerically simulated dependences of strength of water-saturated samples are well described by a logistic function. This bears witness to the decisive role of the competition of two processes, such as pore pressure increase and fluid outflow to the environment, in permeable brittle materials in the course of mechanical loading.

It was shown that strength of a saturated shear band is directly connected with shear rate, fluid viscosity and permeability of the shear band zone and surrounding massif. The governing combination of these parameters $A_{x y}$, together with the obtained empirical dependence $\tau_{c}\left(A_{x y}\right)$ allows prediction of shear band strength under given loading conditions. The latter may be especially important for estimation of transition point of the shear mode of consolidated fault segments from stick to dynamic slip.

The reported results demonstrate broad potentials of the developed DEM-based coupled model of a poroelastic medium and show the importance of numerical modeling application to the study of the mechanical properties (including strength) of dynamically loaded fluid-saturated materials.

\section{References}

1. Volfkovich YuM, Filippov AN, Bagotsky VS (2014) Structural properties of porous materials and powders used in different fields of science and technology. Springer, London

2. Doyen PM (1988) Permeability, conductivity, and pore geometry of sandstone. J Geophys Res-Solid Earth 93(B7):7729-7740

3. Dong T, Harris NB, Ayranci K, Twemlow CE, Nassichuk BR (2015) Porosity characteristics of the Devonian Horn River shale, Canada: insights from lithofacies classification and shale composition. Int J Coal Geol 141-142:74-90

4. Carey JW, Lei Z, Rougier E, Mori H, Viswanathan H (2015) Fracture-permeability behavior of shale. J Unconv Oil Gas Resour 11:27-43

5. Taylor D (2007) Fracture and repair of bone: a multiscale problem. J Mater Sci 42:8911-8918

6. Fernando JA, Chung DDL (2002) Pore structure and permeability of an alumina fiber filter membrane for hot gas filtration. J Porous Mater 9:211-219 
7. Azami M, Samadikuchaksaraei A, Poursamar SA (2010) Synthesis and characterization of hydroxyapatite/gelatin nanocomposite scaffold with controlled pore structure for bone tissue engineering. Int J Artif Organs 33:86-95

8. Wolfram S (1986) Theory and applications of cellular automata. World Scientific Publishing Co., Inc., New Jersey

9. Psakhie SG, Smolin AY, Korostelev SY, Dmitriev AI, Shilko EV, Alekseev SV (1995) Investigation of establishment of steady-state deformation of solids by movable cellular automata method. Pisma Zh Tekh Fiz 21(20):72-76

10. Psakhie SG, Ostermeyer GP, Dmitriev AI, Shilko EV (2000) Method of movable cellular automata as a new trend of discrete computational mechanics. I. Theoretical description. Phys Mesomech 3(2):5-12

11. Psakhie SG, Horie Y, Ostermeyer GP, Korostelev SYu, Smolin AYu, Shilko EV, Dmitriev AI, Blatnik S, Špegel M, Zavšek S (2001) Movable cellular automata method for simulating materials with mesostructure. Theoret Appl Fract Mech 37(1-3):311-334

12. Sukop MC, Thorne DT (2007) Lattice Boltzmann modeling: an introduction for geoscientists and engineers. Springer, Berlin

13. Panin VE (ed) (1998) Physical mesomechanics of heterogeneous media and computer-aided design of materials. Cambridge International Science Publishing Ltd., Cambridge

14. Biot MA (1941) General theory of three-dimensional consolidation. J Appl Phys 12:155-164

15. Biot MA (1957) The elastic coefficients of the theory of consolidation. J Appl Phys 24:594-601

16. Garagash IA, Nikolaevskiy VN (1989) Nonassociative flow rules and localization of plastic deformation. Adv Mech 12(1):131-183 (in Russian)

17. Hamiel Y, Lyakhovsky V, Agnon A (2004) Coupled evolution of damage and porosity in poroelastic media: theory and applications to deformation of porous rocks. Geophys J Int 156(3):701-713

18. Lyakhovsky V, Hamiel Y (2007) Damage Evolution and Fluid Flow in Poroelastic Rock. Izv Phys Solid Earth 43(1):13-23

19. Meirmanov AM (2007) Nguetseng's two-scale convergence method for filtration and seismic acoustic problems in elastic porous media. Sib Math J 48(3):645-667

20. Hörlin NE, Göransson P (2010) Weak, anisotropic symmetric formulations of Biot's equations for vibro-acoustic modelling of porous elastic materials. Int J Numer Meth Eng 84(12):1519_ 1540

21. Hörlin NE (2010) A symmetric weak form of Biot's equations based on redundant variables representing the fluid, using a Helmholtz decomposition of the fluid displacement vector field. Int J Numer Meth Eng 84(13):1613-1637

22. Bocharov OB, Rudiak VI, Seriakov AV (2014) Simplest deformation models of a fluid-saturated poroelastic medium. J Min Sci 50(2):235-248

23. Cundall PA, Strack ODL (1979) A discrete numerical model for granular assemblies. Géotechnique 29(1):47-65

24. Mustoe GGW (1992) A generalized formulation of the discrete element method. Eng Comput 9(2):181-190

25. Shi G-H (1992) Discontinuous deformation analysis-a new numerical model for statics and dynamics of block systems. Eng Comput 9(2):157-168

26. Lisjak A, Grasseli G (2014) A review of discrete modeling techniques for fracturing processes in discontinuous rock masses. J Rock Mech Geotech Eng 6(4):301-314

27. Munjiza AA (2004) The combined finite-discrete element method. Wiley, Chichester

28. Bićanić N (2004) Encyclopedia of computational mechanics. Volume 1: Fundamentals. Wiley, Chichester (Discrete element methods)

29. Jing L, Stephansson O (2007) Fundamentals of discrete element method for rock engineering: theory and applications. Elsevier, Amsterdam

30. Williams JR, Hocking G, Mustoe GGW (1985) The theoretical basis of the discrete element method. NUMETA 1985 Rotterdam-Numerical methods of engineering, theory and applications, pp 897-906 
31. Potyondy DO, Cundall PA (2004) A bonded-particle model for rock. Int J Rock Mech Min Sci 41(8):1329-1364

32. Tavarez FA, Plesha ME (2007) Discrete element method for modelling solid and particulate materials. Int J Numer Meth Eng 70(4):379-404

33. Psakhie SG, Shilko EV, Grigoriev AS, Astafurov SV, Dimaki AV, Smolin AY (2014) A mathematical model of particle-particle interaction for discrete element based modeling of deformation and fracture of heterogeneous elastic-plastic materials. Eng Fract Mech 130:96-115

34. Shilko EV, Psakhie SG, Schmauder S, Popov VL, Astafurov SV, Smolin AY (2015) Overcoming the limitations of distinct element method for multiscale modeling of materials with multimodal internal structure. Comput Mater Sci 102:267-285

35. Hahn M, Wallmersperger T, Kröplin B-H (2010) Discrete element representation of discontinua: proof of concept and determination of material parameters. Comput Mater Sci 50:391-402

36. Dmitriev AI, Osterle W, Kloß H (2008) Numerical simulation of typical contact situations of brake friction materials. Tribol Int 41(1):1-8

37. Psakhie S, Ovcharenko V, Yu B, Shilko E, Astafurov S, Ivanov Y, Byeli A, Mokhovikov A (2013) Influence of features of interphase boundaries on mechanical properties and fracture pattern in metal-ceramic composites. J Mater Sci Technol 29(11):1025-1034

38. Psakhie SG, Ruzhich VV, Shilko EV, Popov VL, Astafurov SV (2007) A new way to manage displacements in zones of active faults. Tribol Int 40(6):995-1003

39. Psakhie SG, Shilko EV, Smolin AYu, Dimaki AV, Dmitriev AI, Konovalenko IS, Astafurov SV, Zavshek S (2011) Approach to simulation of deformation and fracture of hierarchically organized heterogeneous media, including contrast media. Phys Mesomech 14(5-6):224-248

40. Zavšek S, Dimaki AV, Dmitriev AI, Shilko EV, Pezdič J, Psakhie SG (2013) Hybrid cellular automata method. Application to research on mechanical response of contrast media. Phys Mesomech 16(1):42-51

41. Psakhie SG, Dimaki AV, Shilko EV, Astafurov SV (2016) A coupled discrete element-finite difference approach for modeling mechanical response of fluid-saturated porous material. Int J Numer Meth Eng 106(8):623-643

42. Shilko EV, Dimaki AV, Psakhie SG (2018) Strength of shear bands in fluid-saturated rocks: a nonlinear effect of competition between dilation and fluid flow. Sci Rep 8(1):1428

43. Zwietering MH, Jongenburger I, Rombouts FM, van't Riet K (1990) Modeling of the bacterial growth curve. Appl Environ Microbiol 56(6):1875-1881

44. Ben-Zion Y, Sammis C (2010) Mechanics, structure and evolution of fault zones. Birkhauser Verlag AG, Basel

45. Fossen H, Schultz RA, Shipton ZK, Mair K (2007) Deformation bands in sandstone: a review. J Geol Soc 164:755-769

46. Agard P, Augier R, Monie P (2011) Shear band formation and strain localization on a regional scale: evidence from anisotropic rocks below a major detachment (Betic Cordilleras, Spain). J Struct Geol 33(2):114-131

47. Marone C, Scholz CH (1989) Particle-size distribution and microstructures within simulated fault gouge. J Struct Geol 11(7):799-814

48. Marone C (1998) Laboratory-derived friction lows and their application to seismic faulting. Annu Rev Earth Planet Sci 26:643-696

49. Marone C (1995) Fault zone strength and failure criteria. Geophys Res Lett 22(6):723-726

50. Scuderi MM, Carpenter BM, Johnson P, Marone C (2015) Poromechanics of stick-slip frictional sliding and strength recovery on tectonic faults. J Geophys Res: Solid Earth 120(10):6895-6912

51. Duarte JC, Schellart WP, Cruden AR (2015) How weak is the subduction zone interface? Geophys Res Lett 42(8):2664-2673

52. Weiss J, Pellissier V, Marsan D, Arnaud L, Renard F (2016) Cohesion versus friction in controlling the long-term strength of a self-healing experimental fault. J Geophys Res: Solid Earth 121(12):8523-8547 
53. Hubbert MK, Rubey WW (1959) Role of fluid pressure in mechanics of overthrust faulting. Geol Soc Am Bull 70(2):115-166

54. Rice JR (1975) On the stability of dilatant hardening for saturated rock mass. J Geophys Res 80(11):1531-1536

55. Hardebeck JL, Hauksson E (1999) Role of fluids in faulting inferred from stress field signatures. Science 285(5425):236-239

56. Rudnicki JW (2001) Coupled deformation-diffusion effects in the mechanics of faulting and failure of geomaterials. Appl Mech Rev 54(6):483-502

57. Chambon G, Rudnicki JW (2001) Effects of normal stress variations on frictional stability of a fluid-infiltrated fault. J Geophys Res: Solid Earth 106(B6):11353-11372

58. Hamiel Y, Lyakhovsky V, Agnon A (2005) Rock dilation, nonlinear deformation, and pore pressure change under shear. Earth Planet Sci Lett 237(3-4):577-589

59. Paterson MS, Wong TF (2005) Experimental rock deformation-the brittle field. Springer, Berlin

60. Rozhko AY, Podladchikov YY, Renard F (2007) Failure patterns caused by localized rise in pore-fluid overpressure and effective strength of rocks. Geophys Res Lett 34(22). https://doi. org/10.1029/2007g1031696

61. Ougier-Simonin A, Zhu W (2013) Effects of pore fluid pressure on slip behaviors: an experimental study. Geophys Res Lett 40(11):1-6

62. Ougier-Simonin A, Zhu W (2015) Effects of pore pressure buildup on slowness of rupture propagation. J Geophys Res: Solid Earth 120(12):7966-7985

63. Scuderi MM, Collettini C (2016) The role of fluid pressure in induced vs. triggered seismicity: insights from rock deformation experiments on carbonates. Sci Rep 6:24852. https://doi.org/ $10.1038 /$ srep 24852

64. Nur A (1975) A note on the constitutive law for dilatancy. Pure Appl Geophys 113:197-206

65. Germanovich LN, Salganik RL, Dyshkin AV, Lee KK (1994) Mechanisms of brittle fracture of rock with pre-existing cracks in compression. Pure Appl Geophys 143:117-149

66. Main IG, Bell AF, Meredith PG, Geiger S, Touati S (2012) The dilatancy-diffusion hypothesis and earthquake predictability. Geol Soc London Spec Publ 367:215-230

67. Duda M, Renner J (2013) The weakening effect of water on the brittle failure strength of sandstone. Geophys J Int 192(3):1091-1108

68. Matthai SK, Fischer G (1996) Quantitative modeling of fault-fluid-discharge and fault-dilationinduced fluid-pressure variations in the seismogenic zone. Geology 24(2):183-186

69. Brace WF, Martin RJ (1968) A test of the law of effective stress for crystalline rocks of low porosity. Int J Rock Mech Min Sci Geomech Abstr 5(5):415-426

70. Atkinson C, Cook JM (1993) Effect of loading rate on crack propagation under compressive stress in a saturated porous material. J Geophys Res: Solid Earth 98(B4):6383-6395

71. Samuelson J, Elsworth D, Marone C (2011) Influence of dilatancy on the frictional constitutive behavior of a saturated fault zone under a variety of drainage conditions. J Geophys Res: Solid Earth 116(B10). https://doi.org/10.1029/2011jb008556

72. Sibson RS (2003) Thickness of the seismic slip zone. Bull Seismol Soc Am 93(3):1169-1178

73. Zoback MD, Barton CA, Brudy M, Castillo DA, Finkbeiner T, Grollimund BR, Moos DB, Peska P, Ward CD, Wiprut DJ (2003) Determination of stress orientation and magnitude in deep wells. Int J Rock Mech Min Sci 40(7-8):1049-1076

74. Chung SF, Randolph MF, Schneider JA (2006) Effect of penetration rate on penetrometer resistance in clay. J Geotech Geoenviron Eng 132(9):1188-1196

75. Quinn TAC, Brown MJ (2011) Effect of strain rate on isotropically consolidated kaolin over a wide range of strain rates in the triaxial apparatus. In: Proceedings of the fifth international symposium on deformation characteristics of geomaterial, 1-3 Sept 2011, Seoul, Korea. IOS Press, pp 607-613

76. Robinson S, Brown MJ (2013) Rate effects at varying strain levels in fine grained soils. In: Proceedings of the 18th international conference on soil mechanics and geotechnical engineering, Paris, 2-6 Sept 2013, pp 263-266 
Open Access This chapter is licensed under the terms of the Creative Commons Attribution 4.0 International License (http://creativecommons.org/licenses/by/4.0/), which permits use, sharing, adaptation, distribution and reproduction in any medium or format, as long as you give appropriate credit to the original author(s) and the source, provide a link to the Creative Commons license and indicate if changes were made.

The images or other third party material in this chapter are included in the chapter's Creative Commons license, unless indicated otherwise in a credit line to the material. If material is not included in the chapter's Creative Commons license and your intended use is not permitted by statutory regulation or exceeds the permitted use, you will need to obtain permission directly from the copyright holder.

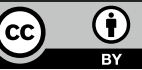

